\title{
Experiências de aprendizagem mais efetivas segundo acadêmicos de Odontologia
}

Fabio Marzullo Zaroni*; Guilherme Strujak*; Gabriel Leonardo Magrin*; Luciana Reichert da Silva Assunção**; Antônio Adilson Soares de Lima**; Ângela Fernandes**

* Aluno do Programa de Pós-Graduação em Odontologia (Mestrado), Departamento de Estomatologia, Universidade Federal do Paraná - UFPR

* Doutor(a), Professor(a) do Departamento de Estomatologia da Universidade Federal do Paraná - UFPR

\section{RESUMO}

O ensino centrado no professor ainda é amplamente abordado na maioria das Instituições de Ensino Superior brasileiras. Este método não prioriza a participação ativa do aluno no processo de construção do conhecimento. O objetivo desta pesquisa foi conhecer as experiências de aprendizagem consideradas mais significativas pelos acadêmicos de odontologia. Foi realizada uma pesquisa quantitativa, com análise descritiva e analítica utilizando questionário previamente testado. A amostra foi composta por 274 acadêmicos, regularmente matriculados no ano de 2014. Do total de alunos, 209 (76,3\%) era do sexo feminino, com média de idade de 21,3 $\pm 2,5$ anos. Os resultados demonstraram que a estratégia de ensino que mais colaborou para a aprendizagem do aluno foram as atividades desenvolvidas em aulas práticas (186, 67,9\%), contribuindo, segundo 171 (92,3\%) alunos, de maneira intensa para sua formação. A segunda estratégia de ensino mais citada foi a participação em projetos de extensão (17, $6,2 \%$ ). Observou-se tendência na preferência pelas atividades extraclasse entre acadêmicos do sexo feminino. Não houve diferença estatisticamente significativa entre o tipo de estratégia de ensino e idade $(\mathrm{p}=0,211)$. Atividades em aulas práticas foram as que mais contribuíram para o aprendizado do acadêmico no âmbito universitário. Espera-se que os resultados deste estudo, em conjunto com as Diretrizes Curriculares Nacionais, contribuam para a discussão sobre a necessidade de melhorias e inovação no ensino em odontologia.

Descritores: Aprendizagem. Ensino. Educação em Odontologia.

\section{INTRODUÇÃO}

A aprendizagem é um processo de mudança de comportamento obtido por meio da experiência construída por fatores emocionais, neurológicos, relacionais e ambientais. Aprender é o resultado da interação entre estruturas mentais e o meio ambiente. A educação como interatividade contempla tempos e espaços novos, diálogo, problematização e produção própria dos educandos. O professor exerce a sua habilidade de mediador das construções de aprendizagem, comunicando, colaborando e exercendo o seu papel de coautor do processo de aprendizagem dos alunos. Nesse enfoque centrado na aprendizagem, o conhecimento é construído e reconstruído continuamente. Todo o processo ensino-aprendizagem deve ser construído com seus objetivos: domínio cognitivo, afetivo e psicomotor $^{1}$.

Ensinar supõe provocar situações que 
levem o aluno a estabelecer o máximo de relações possíveis envolvendo o objeto em estudo, estabelecendo o crescimento intelectual. Aprender significa que o educando, diante de situações novas, é capaz de buscar alternativas, criando suas próprias explicações com autonomia de pensamento. Com as constantes transformações do mundo globalizado e informatizado, o professor já não pode ser considerado o único detentor do saber e que apenas lhe basta transmitir o conhecimento $^{2}$.

Vivemos um processo de esgotamento do modelo tradicional de educação superior. A necessidade de mudanças dessa formação tem estado na pauta das discussões há algum tempo. A questão da formação de profissionais de saúde envolve diretamente as oportunidades advindas do mercado de trabalho, o perfil profissional e a satisfação das demandas populacionais ${ }^{3}$.

Alguns dos problemas enfrentados na formação do profissional de odontologia na atualidade são deficiências quanto aos conteúdos relacionados à formação cultural, humanística e política; o professor universitário privilegiando os saberes técnicos em detrimento dos pedagógicos, ainda com grande força do modelo biomédico de ensino; dificuldade dos acadêmicos em demonstrar sensibilidade social e preocupação com os problemas da população; dificuldades para atuação em saúde coletiva e deficiência no conhecimento das atribuições dos membros da equipe de Saúde da Família ${ }^{4}$.

Deste modo, é imprescindível que a educação superior em odontologia deva assumir a formação de competências para atuar em um contexto mais amplo, preparando o profissional para a construção do seu conhecimento, e conduzindo de maneira contínua em direção a uma formação integral. A interdisciplinaridade se coloca como uma possibilidade de se corrigir distorções provocadas pelo excesso das especializações com fragmentação do conhecimento. A prática atual da profissão é fruto da exaustiva ênfase dada ao caráter individualista que caracteriza a odontologia. A relação existente entre saúde e educação deve se focar na adequação dos profissionais às necessidades sociais ${ }^{3}$.

Esta condição poderá ser obtida quando a formação universitária considerar o sistema de saúde vigente no Brasil, o trabalho em equipe e a ação integral em saúde. Por isso, o real papel da universidade deveria ser o de identificar corretamente os problemas de saúde de cada município ou região e elaborar estratégias de como resolvê-los, direcionando o ensino e a pesquisa para ações de impactos sociais que possibilitem melhores condições de vida para a população ${ }^{5}$.

O desafio está na perspectiva de se desenvolver a autonomia em coalizão com o coletivo. A educação deve ser capaz de desencadear uma visão do todo, da interdependência das disciplinas, com a expansão da consciência individual e coletiva. O estudante deve ser capaz de relacionar o conteúdo apreendido aos conhecimentos prévios, ou seja, o conteúdo novo deve apoiarse em estruturas cognitivas já existentes. Considerando-se, ainda, que a graduação dura somente alguns anos, enquanto a atividade profissional pode permanecer por décadas e que os conhecimentos e competências vão se transformando velozmente, torna-se essencial pensar em uma metodologia para uma prática de educação libertadora, na formação de um profissional ativo e apto a aprender ${ }^{6}$.

\section{MATERIAL E MÉTODOS}

Foi realizada uma pesquisa quantitativa, com análise descritiva utilizando questionário previamente testado. $\mathrm{O}$ projeto de pesquisa foi aprovado pelo Comitê de Ética em Pesquisa do Setor de Ciências da Saúde sob CAAE n. 24819813.7.0000.0102. 
A amostra foi composta por acadêmicos do curso de Odontologia da Universidade Federal do Paraná, de ambos os sexos, matriculados no ano de 2014.

Os critérios de inclusão da amostra foram estar regularmente matriculado no $2^{\circ}$ ao $9^{\circ}$ períodos do curso no primeiro semestre de 2014 e aceitar participar da pesquisa por meio da assinatura do Termo de Consentimento Livre e Esclarecido (TCLE).

Durante o período de matrícula dos cursos de graduação, em fevereiro de 2014, os pesquisadores convidaram os acadêmicos a participar da pesquisa. Os acadêmicos foram informados sobre os objetivos da mesma e aqueles que desejaram participar assinaram o TCLE. Após isso, os acadêmicos receberam um questionário específico para este estudo. Cada questionário foi identificado por um código numérico e não pelo nome do acadêmico, assegurando desta forma a confidencialidade das informações.

O questionário apresentava uma questão objetiva (figura 1) e cada uma das onze possibilidades de respostas foi apresentada de forma individual, com opção de assinalar o grau de contribuição de cada atividade para a aprendizagem do entrevistado: não contribuiu, contribuiu pouco, e contribuiu muito. Para as atividades extracurriculares foi incluída a opção: nunca fiz.

Todas as análises estatísticas foram realizadas com o auxílio do programa SPSS Statistics ${ }^{\mathrm{TM}}$ (SPSS para Windows, versão 20.0, SPSS Inc., Chicago, IL, EUA), considerando um nível de significância 5\%. Foi utilizado o teste Qui-quadrado de Pearson, sendo que as variáveis qualitativas foram descritas por percentuais.

\section{RESULTADOS}

Foram incluídos 274 acadêmicos, sendo $209(76,3 \%)$ do sexo feminino e 65 $(67,9 \%)$ do sexo masculino. A média de idade foi de 21,3 $\pm 2,5$ anos.

As estratégias de ensino que mais colaboraram para a aprendizagem foram a participação em aulas práticas $(186,67,9 \%)$, projeto de extensão $(17,6,2 \%)$ e assistir palestras (jornadas, congressos, simpósios e outros, $16,5,8 \%)$.

Segundo 171 alunos (92,3\%), a participação em aulas práticas contribuiu de maneira intensa para o aprendizado (figura 2).

A partir do teste Qui-quadrado de Pearson, observou-se que não houve diferença estatisticamente significativa entre o tipo de estratégia de ensino escolhida e idade $(\mathrm{p}=0,211)$ (tabela 1).

\section{DISCUSSÃO}

O presente estudo foi realizado com objetivo de avaliar quais as estratégias de aprendizagem mais significativas para os acadêmicos do curso de odontologia da UFPR.

As estratégias de aprendizagem podem ser divididas em dois grandes grupos: estratégias cognitivas e metacognitivas ${ }^{7}$. As estratégias cognitivas auxiliam os estudantes a operar diretamente com a informação, refletindo na forma do estudante organizar, armazenar e elaborar as informações ${ }^{8}$. Já as metacognitivas envolvem, simultaneamente, o estabelecimento de objetivos de estudo (planejamento), o conhecimento sobre a própria compreensão (monitoramento) e o conhecimento de como compreender (regulação) ${ }^{9}$.

A aprendizagem é um processo ativo que deve ser fomentado por meio de métodos que permitam ao aluno o uso da iniciativa, primando pelo seu senso de responsabilidade ${ }^{10}$.

A passagem da consciência ingênua para a consciência crítica, requer a curiosidade criativa, indagadora e insatisfeita de um sujeito ativo, que reconhece a realidade como mutável ${ }^{11,12}$. 


\section{"EXPERIÊNCIAS DE APRENDIZAGEM MAIS EFETIVAS NA PERSPECTIVA DE ACADÊMICOS DE ODONTOLOGIA"}

Código

Circule o(s) período(s) do curso em que você está matriculado no primeiro semestre de 2014.

$$
\begin{array}{llllllll}
2 & 3 & 4 & 5 & 6 & 7 & 8 & 9
\end{array}
$$

Sexo: ( ) Masculino ( ) Feminino Idade......... Data de nascimento ...................

1. Qual a experiência de aprendizagem mais significativa para você, durante o curso de Odontologia? (marcar apenas uma opção)

( ) Assistindo uma aula teórica.

( ) Participando de uma aula prática.

( ) Assistindo uma palestra (jornadas, congressos, simpósio, outros).

( ) Desenvolvendo projeto de pesquisa (Iniciação científica, TCC, outros).

( ) Apresentando algum tema em sala de aula.

( ) Apresentando tema livre em evento científico.

( ) Apresentando painel em evento científico (jornadas, congressos, simpósio, outros).

( ) Participando de um Projeto de Extensão.

( ) Participando de um Grupo PET (Programa de Educação Tutorial).

( ) Utilizando a internet ou outras tecnologias.

( ) Lendo material bibliográfico impresso (artigo, livro, apostila).

Nos quadros abaixo, circule o quanto cada ATIVIDADE CONTRIBUIU PARA SEU APRENDIZADO

1. Aula teórica

Não contribuiu

$\begin{array}{ccc}1 & 2 & 3 \\ \text { Pouco } & & \text { Muito }\end{array}$

2. Aula prática

0

Não contribuiu

3. Assistir uma palestra

0

Não contribuiu

4. Desenvolver um projeto de pesquisa 00

Nunca fiz

Não contribuiu

Não contribuiu

2

Pouco

$\begin{array}{lll}1 & 2 & 3\end{array}$

Muito

5. Apresentar um tema específico em sala de aula 00

Não contribuiu

1

2

Pouco

3

Muito

Nunca fiz

1

Pouco

2

3

Muito 00

Nunca fiz

Não contribuiu

1
Pouco

2

3

Muito

7. Participar de um projeto de extensão 00

$$
0
$$

Pouco

2

Muito

Nunca fiz

Não contribuiu

1

Pouco

2

3

8. Participar de um grupo PET (Programa de Educação Tutorial) 00

Não contribuiu

Pouco

23

Nunca fiz

uxilia (ou contribu)

\section{0}

Não contribuiu

1

Pouco

2

Nunca fiz

10. De que forma a tecnologia (internet) auxilia o seu aprendizado?

( )

$$
\text { ( ) ( ) }
$$

( )

Tradução Pesquisa Leitura Baixar aulas Outros

( )

11. Leitura de material bibliográfico impresso (livro, artigo, apostila, etc)

$0 \quad 112$

Não contribuiu

Pouco

Muito

Figura 1 - Questionário aplicado. 


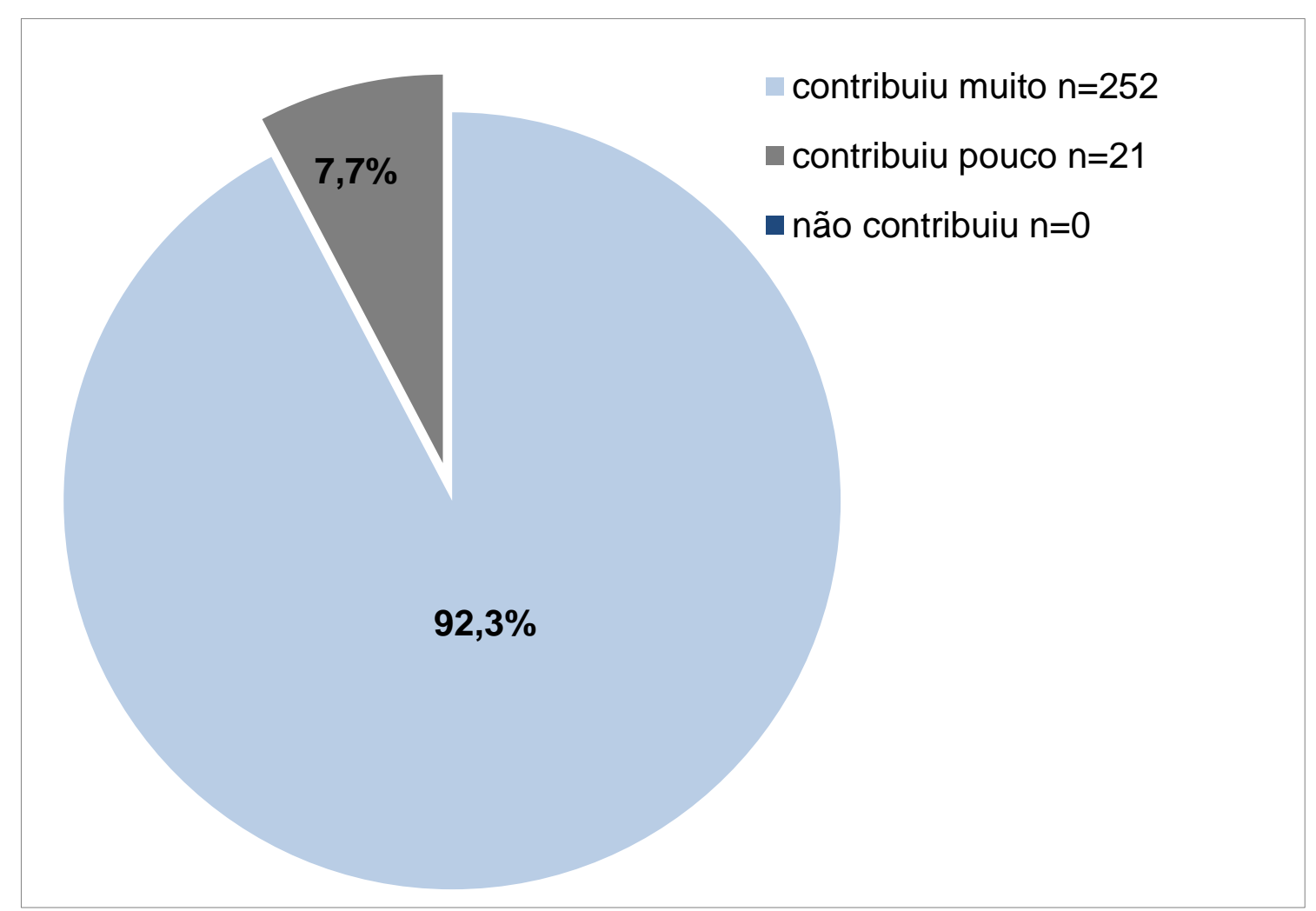

Figura 2 - Contribuição das aulas práticas, segundo as respostas dos entrevistados.

Tabela 1 - Experiências de aprendizagem mais significativas durante o curso de odontologia segundo a idade dos entrevistados.

\begin{tabular}{lcccc}
\hline \multicolumn{1}{c}{ TIPO DE ATIVIDADE } & $\begin{array}{c}\text { Até } 21 \text { anos } \\
\mathrm{n}\end{array}$ & $\begin{array}{c}\text { Mais de } 21 \text { anos } \\
\mathrm{n}\end{array}$ & $\begin{array}{c}\text { Total } \\
\mathrm{n}\end{array}$ & $p^{*}$ \\
\hline $\begin{array}{l}\text { Atividade em sala de aula ou } \\
\text { clínica }\end{array}$ & 128 & 83 & 211 & \\
$\begin{array}{l}\text { Participação de congressos e afins } \\
\text { Atividade extra-classe }\end{array}$ & 13 & 5 & 18 & 0,211 \\
Material bibliográfico e internet & 12 & 15 & 27 & \\
\hline Total & 9 & 9 & 18 & \\
\hline
\end{tabular}

A educação contemporânea deve pressupor um discente capaz de auto gerenciar ou autogovernar seu processo de formação ${ }^{6}$.
De acordo com os resultados obtidos neste estudo, para $186(67,9 \%)$ dos alunos, a atividade mais significativa e que mais contri- 
bui para a aprendizagem foi o treinamento em aula prática. Este resultado é muito provável pela característica técnica do treinamento profissional da odontologia. Há também, desde o início da odontologia a valorização da prática e especialização em detrimento do estudo das ciências básicas de do conhecimento amplo.

Araújo et al. $(2014)^{13}$ em pesquisa também tiveram como resultado, apoio dos acadêmicos à aula prática, parecendo aumentar o interesse dos alunos e agregando mais conhecimento.

Para $17(6,2 \%)$ dos acadêmicos participantes do presente estudo, projetos de extensão foram as atividades que mais contribuíram para o aprendizado. Verifica-se aqui uma pequena porcentagem dos alunos se engajando num dos maiores objetivos da universidade que é extrapolar o conhecimento construído para o benefício da população.

A inclusão dos alunos da área de saúde no atendimento extramuros junto as equipes do Programa de Saúde da Família traz benefícios ao aprendizado, e simultaneamente percebe-se ocorrer uma troca de experiências e conhecimentos mútuos. Além de que, familiarizam e capacitam os estudantes a trabalhar na realidade que enfrentarão no mercado de trabalho ${ }^{14}$.

Percebe-se que alguns alunos relutam em assumir o perfil necessário para trabalhar em comunidades carentes e sob condições muitas vezes adversas para o bom desempenho da técnica apresentada e executada dentro da Universidade. Além disso, nota-se também a resistência por parte de alguns docentes em assimilar o trabalho inter e multidisciplinar como instrumento mais adequado de formação acadêmica $^{13}$.

Segundo o estudo de Araújo et al. $(2014)^{13}$ as estratégias de ensino mais utilizadas pelos docentes baseiam-se em exposições orais e o método de avaliação consta de avaliações tradicionais (teóricas e práticas), na maioria das vezes objetivas, limitando as possibilidades do aluno demonstrar de que maneira assimilou um tema. Na pesquisa os autores também demonstraram que os acadêmicos avaliaram a aula expositiva dialogada como sendo mais eficiente no processo de aprendizagem. Estudo de Fadel e Baldani (2013) ${ }^{15}$ revelou que 59\% dos acadêmicos pesquisados observam que a metodologia pedagógica baseada na transmissão de informações, centrada na figura do professor ainda é mais utilizada na maioria das disciplinas.

A exposição apresenta algumas vantagens, como a economia de tempo, mas apresenta algumas limitações, como à ênfase na comunicação verbal, autoritarismo do professor e inibição da participação do aluno ${ }^{2}$.

Por este motivo, fica nítida a necessidade de novos modelos pedagógicos que promovam a capacidade criativa dos estudantes. Alguns dos componentes do processo educativo, importantes para desenvolver a capacidade criativa, são: a atitude criativa do professor, criação e uso de estratégias pedagógicas e didáticas nas aulas, promoção de atmosferas criativas e o consequente surgimento da criatividade como valor cultural ${ }^{16}$.

Estratégias de ensino-aprendizagem nas quais o aluno transforma-se em sujeito ativo da aprendizagem, investigando, criticando e desenvolvendo a independência intelectual, como: análise de periódicos, discussão de casos clínicos, seminários, sendo o professor o facilitador do processo, vêm sendo utilizadas pelos docentes em substituição às aulas expositivas. Estimular intelectualmente o aluno exige fazê-lo romper com explicações dos outros, provocando para que ele busque as suas próprias, ou seja, desafiá-lo à autonomia do pensamento ${ }^{2}$.

Assim, é imprescindível que exista uma capacitação do docente, passando por um processo de revisão de conceitos educativos, 
permitindo ao estudante se tornar um agente ativo no processo de ensino, com visão crítica em relação à sua prática profissional ${ }^{3}$.

Para que ocorram essas mudanças na formação dos profissionais da saúde, a estrutura institucional deveria subsidiar cursos de capacitação didático-pedagógica aos seus docentes $^{2}$. São necessárias mudanças e inovações para que seja possível formar profissionais generalistas, humanistas, críticos e reflexivos ${ }^{13}$.

As demandas da saúde coletiva devem ser o eixo que orientam a estruturação curricular e a formação de um profissional apto a interferir na realidade do indivíduo e da comunidade. Estudantes e professores devem ser sujeitos dessa mudança, constituindo um processo coletivo de reflexão crítica sobre as práticas tradicionais de aquisição de novos conhecimentos ${ }^{3}$.

Métodos de aprendizado ativos que acompanhem a ciência emergente, a capacitação do estudante para a busca do seu próprio conhecimento devem ser incentivados $^{17,18,19}$.

É preciso instituições de ensino determinadas, com liderança forte e o auxílio das organizações governamentais competentes para originar as ideias necessárias para alcançar as melhorias e inovações no ensino em odontologia.

\section{CONCLUSÕES}

Atividades em aulas práticas foram as que mais contribuíram para o aprendizado do acadêmico de Odontologia no âmbito universitário. Espera-se que os resultados deste estudo, juntamente com as Diretrizes Curriculares Nacionais, auxiliem a reflexão de coordenadores de curso de odontologia sobre a necessidade de aperfeiçoamento do seu corpo docente em relação aos métodos de ensinoaprendizagem.

\section{REFERÊNCIAS}

1. Demo, P. Política social do conhecimento. Amélia Hamze Colunista Brasil Escola Disponível em: http://educador.brasilesco la.com/trabalho-docente/o-que-e-aprendi zagem.htm

2. Lazzarin HC, Nakama L, Júnior LC. O papel do professor na percepção dos alunos de odontologia. Saúde e Sociedade 2007;16(1):90-101.

3. Araujo ME. Palavras e silêncios na educação superior em odontologia. Ciênc Saúde Coletiva 2006;11(1):179-82.

4. Guimarães FAF, Mello ALSF, Pires ROM. Formação Profissional em Odontologia: Revisão de Literatura. Rev Saúde Pública Santa Catarina 2015;7(3):75-87.

5. Garbin CAS, Saliba NA, Moimaz SAS, Santos KT, O papel das universidades na formação de profissionais na área de saúde. Rev ABENO. 2006;6(1):6-10.

6. Mitre SM, Siqueira-Batista R, Girardi-deMendonça JM, Morais-Pinto NM de, Meirelles C de AB, Pinto-Porto C, et al. Metodologias ativas de ensinoaprendizagem na formação profissional em saúde: debates atuais. Ciênc Saúde Coletiva 2008;13(2):2133-44.

7. Boruchovitch E, Santos AAA, Costa ER, Neves ERC, Cruvinel M, Primi R, et al. A construção de uma escala de estratégias de aprendizagem para alunos do ensino fundamental. Psicologia: Teoria e Pesquisa 2006;22(3):297-304.

8. Dembo MH. Applying educational psychology. $5^{\text {th }}$ ed. New York: Longman; 1994.

9. Oliveira KL, Boruchovitch E, Santos AAA. Estratégias de aprendizagem e desempenho acadêmico: evidências de validade. Psicologia: Teoria e Pesquisa 2009;25(4):531-6. 
10. Almeida LS. Facilitar a aprendizagem: ajudar os alunos a aprender e a pensar. Psicologia escolar e educacional. 2002;6(2):155-65.

11. Freire P. Educação e mudança. São Paulo: Paz e Terra; 1999.

12. Freire P. Pedagogia da autonomia: saberes necessários à prática educativa. $33^{\mathrm{a}} \mathrm{ed}$. São Paulo: Paz e Terra; 2006.

13. Araújo RJG, Figueiredo LBA, Ribeiro RR. Conhecimento de estratégias pedagógicas no processo ensinoaprendizagem dos alunos do curso de Odontologia. Full Dent Sci 2014;5 (19):464-71.

14. Mendes RF, Moura MS, Prado Júnior RR, Moura LFAD, Lages GP, Gonçalves MPR. Contribuição do Estágio Supervisionado da UFPI para formação humanística, social e integrada. Rev ABENO 2006;6(1):61-5.

15. Fadel CB, Baldani MH. Percepções de formando do curso de odontologia sobre as diretrizes curriculares nacionais. Trab Educ Saúd 2013;11(2):339-354.

16. Klimenko O. La creatividad como un desafío para la educación del siglo XXI. Educación y Educadores. 2009;11(2):191210.

17. Edwards PC. The future of dental education: toward disruptive innovation or incremental improvements? Oral Surg Oral Med Oral Pathol Oral Radiol 2015;119(3):257-9.

18. Hupp J. Innovation in dental education: empty buzzword or real movement? Oral Surg Oral Med Oral Pathol Oral Radiol Endod 2008;105(1):1-4.

19. Freeman S, Eddy SL, McDonough M, Smith MK, Okoroafor N, Jordt H, Wenderoth MP. Active learning increases student performance in science, engineering, and mathematics. Proc Natl Acad Sci USA 2014;111(23):8410-5.

\section{ABSTRACT \\ Most effective learning experiences for dental students}

The teacher-centered method is still widely used in most universities in Brazil. This method does not prioritize the active participation of the student in the knowledge construction process. The objective of this research was to identify the most significant learning experience according dental students. A quantitative study, with descriptive and analytical analysis using pre-tested questionnaire was conducted. Of the total students, $209(76.3 \%)$ were female, with a mean age of $21.3 \pm 2.5$ years. The results showed that the teaching strategy that most contributed to student learning activities were developed in practical classes, 186 (67.9\%), contributing for $171(92.3 \%)$ students, intensively for their training. The second most cited teaching strategy by dental students was to participate in extension projects $(17 / 6.2 \%)$. There was a trend in preference for extracurricular activities among female dental students. There was no statistically significant difference between learning experience preference and age $(p=0.211)$. Activities in practical lessons were the ones that most contributed to learning for dental students. It is expected that the results of this study, together with the National Curriculum Guidelines could contribute in the discussion about the need of improvement and innovation in dental education.

Descriptors: Learning. Teaching. Dental Education.

Correspondência para:

Profa. Dra. Ângela Fernandes

e-mail: angelfnandes@ hotmail.com

Avenida Prefeito Lothário Meissner, 632 Jardim Botânico

80210-170 - Curitiba - Paraná 